Revista Perspectivas Online: Biológicas \& Saúde Agosto de 2019, Vol.9, n 30, p.13-23 ISSN: 2236-8868 (Online)

DOI: $10.25242 / 886893020191736$

\title{
PERCEPÇÃO DE DISCENTES DE EDUCAÇÃO FÍSICA SOBRE A IMAGEM CORPORAL DE MULHERES ADULTAS
}

Laís Carolina Fernandes ${ }^{1}$, Renato Melo Ferreira ${ }^{1}$ \& Everton Rocha Soares ${ }^{1 *}$,

\section{RESUMO}

FERNANDES, L.C.; FERREIRA, R.M. \& SOARES, E.R. Percepção de discentes de educação física sobre a imagem corporal de mulheres adultas. Perspectivas Online: Biológicas \& Saúde. v. 9, n 30, p.13-23, 2019.

O objetivo foi verificar o nível de percepção subjetiva de discentes ingressantes e concluintes do curso de Educação Física da Universidade Federal de Ouro Preto sobre a imagem corporal, massa corporal e percentual de gordura de mulheres adultas. Participaram do estudo 26 discentes, de ambos os sexos (25,0 $\pm 6,6$ anos), matriculados no curso de Bacharelado em Educação Física; e dez voluntárias (23,5 4,4 anos) não estudantes do curso de Educação Física. As dez voluntárias atuaram como modelos para obtenção de fotografias de corpo inteiro e tiveram medidas a massa corporal e o percentual de gordura estimado. Os discentes de Educação Física, a partir das fotografias das modelos, estimaram a imagem corporal, massa corporal e o percentual de gordura das voluntárias. Nossos resultados mostraram que discentes ingressantes e concluintes tiveram percepção semelhante entre eles sobre a imagem corporal, massa corporal e percentual de gordura, avaliados a partir das fotografias das modelos. Porém os valores de massa corporal medidos nas mesmas foram menores do que os indicados pelos discentes. Acreditase que o processo de formação no curso de Educação Física por si só, não influencia na percepção da imagem corporal, massa corporal e percentual de gordura de mulheres adultas.

Palavras-chave: Percepção Corporal; Fotografias; Estudantes; Variáveis Antropométricas. 


\begin{abstract}
The objective of the present study was to verify the level of subjective perception of freshmen and veteran students of the Physical Education course of the Federal University of Ouro Preto on body image, body mass and body fat of adult women. Twenty-six students of both sexes $(25.0 \pm 6.6$ years) enrolled in the bachelor's degree in Physical Education participated in the study; and ten women (23.5 \pm 4.4 years), not students of the Physical Education course. The ten volunteers acted as models to obtain full body photographs and had measured body mass and body fat. The

students of Physical Education, based on the photograph of the models, estimated the body image, body mass and body fat of the women. Our results showed that freshmen and veteran students had similar perceptions of body image, body mass and body fat evaluated from photographs. However, the values of body mass measured in those women were smaller than those indicated by the students. It is believed that the graduate process in the Physical Education course alone does not influence the perception of body image, body mass and body fat of adult women.
\end{abstract}

Keywords: Body Perception; Photographs; Students; Anthropometric Measures.

\footnotetext{
${ }^{1}$ Universidade Federal de Ouro Preto (UFOP) - Grupo de Estudos e Pesquisas em Avaliação e Musculação - GEPAM -

Rua Professor Paulo Magalhães Gomes, 122 - Bauxita, Ouro Preto - MG, 35400-000 - Brasil

(*)e-mail: everton@ufop.edu.br

Data de recebimento: 28/05/2019 Aceito para publicação: 15/07/2019
} 


\section{INTRODUÇÃO}

A imagem corporal (IC) é entendida como um sistema multidimensional que descreve amplamente as representações internas e externas da estrutura corporal e da aparência física, resultando na representação mental que as pessoas têm quanto ao tamanho e forma do próprio corpo ou de outras pessoas (KAY, 1996; DAVISON e MCCABE, 2005; DAMASCENO et al., 2006).

A preocupação com a aparência física é algo inerente a sociedade desde as civilizações antigas, onde se sentir fisicamente aceito, reflete reconhecer-se dentro de um determinado padrão de beleza imposto e a ser seguido (BARRET et al., 2002). No entanto, a preocupação excessiva pelo corpo considerado "perfeito" pode favorecer a ocorrência de transtornos associados à IC como vigorexia (AZEVEDO et al., 2016; FLORIANO e D'ALMEIDA, 2016), bulimia (FISHER et al., 2019) e anorexia (MARTINS et al., 2010). Desta forma, quanto mais uma pessoa concentra-se em sua aparência corporal, pior ela tende a se sentir a respeito do que vê, encontrando defeitos imperceptíveis ou que as vezes nem existem (FERREIRA et al., 2005; GIORDANI, 2006).

Diferentes estudos têm avaliado o nível de satisfação dos indivíduos com a própria IC (DAMASCENO et al., 2005; MARTINS et al., 2010; FISHER et al., 2019). De forma geral, tem-se observado que tanto os homens quanto as mulheres estão insatisfeitos com a própria IC (DAVISON e MCCABE, 2005; ASSUMPÇÃO et al., 2010). No entanto, de forma geral, observa-se que as mulheres são mais insatisfeitas com a própria IC do que os homens (GARNER et al., 1980; DAVISON e MCCABE, 2005; ALGARS et al., 2009; FERRARI et al. , 2012).

Diante da situação de descontentamento com a IC, homens e mulheres procuram se engajar na prática regular de exercícios físicos como forma de combater essa insatisfação (FERREIRA et al., 2005; MEDEIROS et al., 2017). O Profissional de Educação Física, por sua vez, tem como objeto de estudo e de aplicação a motricidade humana, a cultura do movimento corporal, com foco nas diferentes formas e modalidades do exercício físico, ginástica, jogo, esporte, lutas e dança, visando atender às necessidades sociais no campo da saúde, educação e formação, cultura, alto rendimento esportivo e do lazer (BRASIL, 2018). Nesse sentido, acredita-se que o profissional de Educação Física deva também compreender os diferentes aspectos inerentes à IC e os distúrbios a ela relacionados, de forma que em sua prática profissional possa lidar melhor com as insatisfações de seus clientes (FERNANDES et al., 2005) e o eterno objetivo de alcançar um corpo "perfeito".

Silva et al. (2018) identificaram que ingressantes e concluintes do curso de Bacharelado em Educação Física da Universidade Federal de Ouro Preto, a partir da análise de fotografias de homens adultos, têm percepção semelhante quanto a IC e massa corporal dos indivíduos fotografados. Entretanto, a percepção do percentual de gordura indicada a partir da análise de fotografias, mostrou que ingressantes e concluintes tem percepção diferente quanto a esse parâmetro. Uma vez que os valores indicados pelos concluintes foram mais assertivos do que os indicados pelos iniciantes. No entanto, ainda não se sabe se resultados semelhantes ocorreriam caso os indivíduos avaliados fossem do sexo feminino, uma vez que nas mulheres adultas, de forma geral, a distribuição dos tecidos corporais adiposo e muscular é diferente da dos homens adultos (BORKAN e HULTS, 1983; LOPES et al., 1995).

Dessa forma, o objetivo desse estudo foi verificar o nível de percepção subjetiva de discentes ingressantes e concluintes do curso de Educação Física da Universidade Federal de Ouro Preto (UFOP) sobre a IC, massa corporal e percentual de gordura de mulheres adultas.

\section{METODOLOGIA}

Este estudo é de caráter qualitativo e com corte transversal, sendo aprovado pelo comitê de ética em pesquisa com seres humanos da UFOP (Parecer 781.351). 
A amostra desse estudo foi de conveniência e composta por 36 voluntários. Do total amostral 26 eram discentes, de ambos os sexos, do curso de bacharelado em Educação Física da UFOP (25,0 \pm 6,6 anos), denominado grupo de avaliadores (G-AV). Este grupo foi, posteriormente, dividido em G-AV1 ( $n=13)$ e G-AV2 $(n=13)$, composto por alunos matriculados até o $2^{\circ}$ período e alunos dos $7^{\circ}$ e $8^{\circ}$ período, respectivamente. O restante da amostra foi constituído por 10 mulheres jovens (23,5 \pm 4,4 anos), não estudantes do curso de Educação Física, que compuseram o grupo das modelos, denominado G-MOD. No G-MOD foram realizadas medidas da massa corporal, estatura, percentual de gordura e fotografias de corpo inteiro. Para este grupo, também foi avaliada a percepção da auto imagem corporal e a satisfação com a mesma.

Para a medida da massa corporal e da estatura do G-MOD foi utilizado, respectivamente, uma balança digital (Filizola ${ }^{\circledR}$, São Paulo) com estadiômetro acoplado (precisão de $100 \mathrm{~g}$ e $0,5 \mathrm{~cm}$, respectivamente). A determinação da densidade corporal foi feita utilizando um adipômetro científico (Cescorf ${ }^{\circledR}$, Porto Alegre) a partir do método de sete dobras cutâneas, descrito por Jackson, Pollock e Ward (1980) para mulheres. Para conversão da densidade corporal em percentual de gordura, foi utilizada a equação de Siri (1961). Todas as medidas antropométricas foram realizadas por um avaliador experiente e independente ao estudo.

Para avaliação da IC foi utilizado o conjunto de nove silhuetas para mulheres, proposto por Stunkard et al. (1983) (Figura 1).
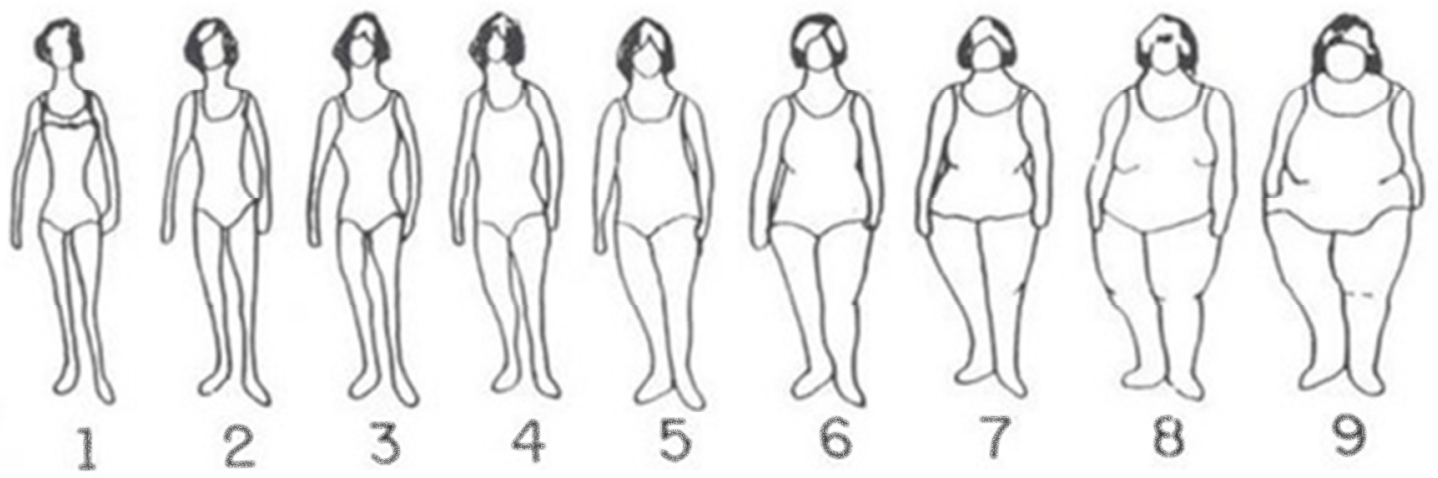

Figura 1: Conjunto de silhuetas proposto por Stunkard et al. (1983).

Para obtenção das fotografias de corpo inteiro das voluntárias do G-MOD foi utilizada uma câmera digital (Sony, modelo W350; 14.1 megapixels), sobre tripé de alumínio (Light Weight, modelo SL-2111). A câmera digital ficou posicionada verticalmente, a um metro e vinte centímetros de altura, afastada a uma distância de três metros da parede, para que pudesse enquadrar a voluntária de corpo inteiro e sem que houvesse distorção no foco da imagem. Nesta parede havia afixada uma escala de estatura (Figura 2). As voluntárias ficaram em posição ortostática, de costas para a escala de estatura, com olhar ao horizonte, trajando apenas um short e um top na cor preta. Após os rostos terem sido ocultados, as 10 fotografias foram reveladas e identificadas aleatoriamente com uma letra de "A" a "J". 




Figura 2: Ilustração do procedimento para obtenção das fotografias.

Após a avaliação das variáveis antropométricas, e obtenção das fotografias de corpo inteiro, foi apresentado individualmente a cada voluntária do G-MOD o conjunto de silhuetas proposto por Stunkard et al. (1983). O objetivo foi avaliar a autoimagem corporal dessas voluntárias e o grau de satisfação para coma IC. Para isso as seguintes perguntas foram feitas: 1) Qual é a silhueta que melhor representa a sua aparência física atualmente, silhueta atual? 2) Qual é a silhueta que você gostaria de ter, silhueta ideal? O pesquisador orientou cada uma das voluntárias acerca do que exatamente era perguntado.

Posteriormente à coleta de dados do G-MOD, os voluntários do G-AV1 e G-AV2, individualmente em uma sala isolada, analisaram e avaliaram as fotografias do G-MOD. Para isso, as dez fotografias foram colocadas em envelopes iguais e sem qualquer marcação. Os envelopes foram embaralhados e expostos separadamente sobre uma mesa. Em seguida os procedimentos da coleta eram lidos para o(a) voluntário(a) do G-AV (1 e 2).

Constava nos procedimentos que o voluntário do G-AV (1 e 2) deveria escolher um envelope por vez, retirar a fotografia e analisá-la durante 10 segundos, utilizando assim da memória de curto prazo, para que a memória de uma foto não influenciasse nas seguintes (COWAN, 2008). Em seguida, o conjunto de silhuetas era mostrado ao indivíduo e realizava-se as seguintes perguntas: 1) Qual a silhueta que melhor representa a fotografia escolhida? 2) Qual é a massa corporal (peso) do indivíduo na fotografia escolhida? 3) Qual o percentual de gordura do indivíduo na fotografia escolhida? $O$ avaliado tinha até 60 segundos para responder as perguntas e os resultados eram anotados em formulário próprio. $\mathrm{O}$ mesmo procedimento ocorreu até que as dez fotografias fossem analisadas e avaliadas. Para não influenciar as respostas dos voluntários dos grupos G-AV1 e G-AV2, nenhuma informação adicional era dada sobre as perguntas supracitadas.

Para a análise dos dados, foi considerada a média dos valores da IC, massa corporal e percentual de gordura atribuída pelos avaliadores G-AV1 e G-AV2.

Visando avaliar a confiabilidade dos resultados obtidos pela avaliação dos grupos G-AV1 e G-AV2, quatro a sete dias após a coleta dos dados, repetiu-se a análise e avaliação das fotografias com onze discentes que se voluntariaram pra tal (seis no G-AV1 e cinco no G-AV2) escolhidos por sorteio. Os coeficientes de correlação intraclasse encontrados foram: Foto silhueta [(G-AV1: $\mathrm{r}=$ $0,98(\mathrm{IC} 95 \%=0,96-0,99) ; \mathrm{G}-\mathrm{AV} 2: \mathrm{r}=0,92(\mathrm{IC} 95 \%=0,71-0,98)]$; Massa corporal [(G-AV1: $\mathrm{r}$ $=0,93(\mathrm{IC} 95 \%=0,73-0,98) ;$ G-AV2: $\mathrm{r}=0,92(\mathrm{IC} 95 \%=0,69-0,98)] \mathrm{e}$; Percentual de gordura [(G-AV1: $r=0,86 ;$ G-AV2: $r=0,94($ IC95\% $=0,75-0,98)]$.

Os dados foram apresentados em média \pm desvio padrão. Para avaliação da distribuição da normalidade dos dados, foi utilizado o teste de D’Agostino \& Pearson. Para avaliação do nível de 
satisfação com a imagem corporal do G-MOD foi utilizado o teste t pareado. Para comparação entre os valores medidos e as avaliações subjetivas dos grupos G-AV1 e G-AV2, foi utilizado o teste de ANOVA para medidas repetidas. Para avaliação da reprodutibilidade das avaliações feitas pelo GAV1 e G-AV2, foi utilizado o coeficiente de correlação intraclasse (Pearson ou Spearman, quando necessário). Todas as análises foram feitas no software estatístico Graphpad Prism versão 5.0, sendo adotado nível de significância de 5\%.

\section{RESULTADOS}

Ao avaliarmos a satisfação das voluntárias do G-MOD coma sua IC atual, observamos que as mesmas se encontravam satisfeitas (Tabela 1).

Tabela 1: Comparação entre a silhueta atual e desejada do G-MOD $(n=10)$

\begin{tabular}{llc}
\hline & IC atual & IC desejada \\
\hline Silhueta proposta por Stunkard et al. & $3,6 \pm 1,4$ & $3,0 \pm 1,0$ \\
\hline
\end{tabular}

Valores em Média \pm Desvio Padrão. IC = Imagem corporal; IC atual = Percepção sobre a autoimagem corporal (STUNKARD et al., 1983) das voluntárias modelos (G-MOD); IC desejada = Imagem corporal desejada pelas voluntárias do G-MOD (STUNKARD et al., 1983). Teste T pareado $(\mathrm{t}=0,138 ; \mathrm{p}=0,89)$.

Ao analisarmos a percepção dos discentes do G-AV1 e G-AV2, sobre a IC nas fotografias do G-MOD, não foram observadas diferenças entre as comparações feitas pelos discentes iniciantes e concluintes do curso de Bacharelado em Educação Física. No entanto, os valores de IC indicados pelo G-AV1 e G-AV2 foram maiores que os indicados pelas voluntárias do G-MOD na sua autoavaliação (Tabela 2).

Tabela 2: Comparação entre a autoavaliação da imagem corporal (IC) feita pelo G-MOD e a percepção dos discentes do G-AV1 e G-AV2 sobre a IC, a partir das fotografias do G-MOD

\begin{tabular}{cccc}
\hline IC & G-MOD & G-AV1 & G-AV2 \\
\hline Silhueta proposta por Stunkard et al. & $3,6 \pm 1,3$ & $4,7 \pm 1,0^{*}$ & $4,6 \pm 1,0^{*}$ \\
& & & \\
\hline
\end{tabular}

Valores em Média \pm Desvio Padrão. IC G-MOD= Autoavaliação da Imagem Corporal (STUNKARD et al., 1983) feita pelas voluntárias do G-MOD ( $\mathrm{n}=10)$; GAV1 = Grupo composto por discentes ingressantes do curso de Bacharelado em Educação Física (n=13); G-AV2 = Grupo composto por discentes concluintes do curso de Bacharelado em Educação Física (n=13). *p<0,05 em comparação com IC G-MOD. Anova para medidas repetidas.

A Tabela 3 apresenta o valor médio da massa corporal das voluntárias do G-MOD, obtido através da avaliação antropométrica e os valores da análise subjetiva do mesmo parâmetro, 
realizada pelos grupos G-AV1 e G-AV2 a partir de fotografias de corpo inteiro do G-MOD. Observamos que os discentes ingressantes e concluintes do curso de Educação Física têm percepção semelhante quanto a massa corporal analisadas a partir das fotografias do G-MOD, porém, os valores de massa corporal indicados pelos discentes (G-AV1 e G-AV2) foram maiores do que os valores medidos no G-MOD.

Tabela 3: Comparação entre a massa corporal (Kg) medida no G-MODe a percepção dos discentes do G-AV1 e G-AV2 sobre a massa corporal, a partir das fotografias do G-MOD

\begin{tabular}{|c|c|c|c|}
\hline & Valores medidos & G-AV1 & G-AV2 \\
\hline Massa corporal & $59,9 \pm 9,2$ & $65,4 \pm 5,3^{*}$ & $64,9 \pm 5,0 *$ \\
\hline
\end{tabular}

Comparando a percepção dos voluntários do G-AV1 e G-AV2 sobre o percentual de gordura nas fotografias e o percentual de gordura estimado no G-MOD, não foram encontradas diferenças significativas (Tabela 4).

Tabela 4: Comparação entre o percentual (\%) de gordura estimado no G-MOD e a percepçãodos discentes do G-AV1 e G-AV2 sobre o \% de gordura, a partir das fotografias do G-MOD

\begin{tabular}{|c|c|c|c|}
\hline & Valor estimado & G-AV1 & G-AV2 \\
\hline$\%$ de gordura & $26,2 \pm 4,9$ & $26,7 \pm 4,1$ & $24,8 \pm 3,3$ \\
\hline \multicolumn{4}{|c|}{$\begin{array}{l}\text { Valores em Média } \pm \text { Desvio Padrão. Valor estimado = valor obtido por meio do método de sete } \\
\text { dobras cutâneas (JACKSON, POLLOCK e WARD, 1980). G-AV1 = Grupo composto por } \\
\text { discentes iniciantes do curso de Bacharelado em Educação Física }(\mathrm{n}=13) \text {. G-AV2 = Grupo } \\
\text { composto por discentes concluintes do curso de Bacharelado em Educação Física (n=13). G- } \\
\text { MOD = Grupo composto por voluntárias modelos }(\mathrm{n}=10) \text {. ANOVA para medidas repetidas. }\end{array}$} \\
\hline
\end{tabular}

\section{DISCUSSÃO}

O presente estudo teve como objetivo verificar o nível de percepção subjetiva de discentes ingressantese concluintes (G-AV1 e G-AV2, respectivamente) do curso de Bacharelado em Educação Física da UFOP sobre a IC, massa corporal e percentual de gordura de mulheres adultas, a partir de fotografias de corpo inteiro. De forma geral, observamos que, a partir da análise de fotografias das mulheres do G-MOD, tanto os discentes iniciantes (G-AV1) quanto os concluintes 
(G-AV2) tiveram percepção semelhante em relação a IC e a massa corporal daquelas mulheres. Entretanto, os valores de IC indicados pelos discentes iniciantes e concluintes foram maiores aos autodeclarados pelas mulheres do G-MOD. Adicionalmente, os valores de massa corporal subjetivados pelos discentes iniciantes e concluintes foram maiores do que os valores de massa corporal medidos no G-MOD. Já os valores do percentual de gordura subjetivados pelo G-AV1 e GAV2 e os medidos no G-MOD foram semelhantes entre si.

De forma geral, as mulheres estão mais insatisfeitas com a própria IC do que os homens, desejando outra como ideal (ALGARS et al., 2009; FERRARI et al., 2012; MEDEIROS et al., 2017). Essa insatisfação parece estar relacionada a questões socioculturais e midiáticas, que "propõem" um padrão definido de corpo perfeito, sendo para os homens o corpo mais forte e volumoso e para as mulheres a magreza, uma forma de aceitação social (GARNER et al., 1980; CASH e GREEN, 1986; ASSUMPÇÃO et al., 2010; FLORIANO, 2016). Nossos resultados apontam que as voluntárias do G-MOD se mostraram, em média, satisfeitas com a sua IC. É possível que a satisfação observada nas voluntárias do G-MOD, esteja relacionada com o tamanho amostral deste grupo $(n=10)$. No entanto sabe-se que diferentes fatores sensoriais, como a recepção e integração de estímulos visuais, táteis e sinestésicos, e fatores não sensoriais, como aspectos cognitivos, afetivos e sociais podem interferir na percepção da imagem corporal, levando a superestimação ou subestimação (CRAWFORD e CAMPBELL, 1999; KAMEL e MCNEILL, 2000; RAMIREZ e ROZEN, 2001) da IC.

Por outro lado, ao compararmos a percepção do G-AV1 e G-AV2 sobre a IC avaliada a partir de fotografias de mulheres adultas, não encontramos diferenças significativas entre os valores indicados. De forma semelhante, Silva et al. (2018) também não observaram diferenças significativas entre a avaliação da IC feita por discentes iniciantes e concluintes do mesmo curso, a partir da avaliação de fotografias de homens adultos. Adicionalmente, observa-se que os valores da autoavaliação da IC feita pelo G-MOD foram significativamente menores do que os indicados pelo G-AV1 e G-AV2. Em conjunto esses resultados sugerem que o nível de formação no curso de Educação física parece não influenciar diretamente na percepção da IC de mulheres e homens adultos, a partir da análise de fotografias de corpo inteiro. Adicionalmente, acredita-se que o fato de os valores de autoavaliação da IC (pelo G-MOD) terem sido diferente dos indicados pelos avaliadores discentes (G-AV1 e G-AV2) possa estar relacionado a fatores intrínsecos e multidimensionais que descrevem de maneira ampla as representações internas da estrutura corporal e da aparência físicado indivíduo, em relação a si mesmo e aos outros, podendo, assim, ser diferente entre diferentes pessoas (KAY, 1996; MCCABE et al., 2006).

No presente estudo as análises referentes a massa corporal mostraram, não haver diferença entre a percepção desse parâmetro antropométrico entre os discentes do G-AV1 e G-AV2. Por outro lado, os valores de massa corporal indicados pelos estudantes de Educação Física foram menores do que os valores medidos das mulheres do G-MOD. Já no estudo de Silva et al. (2018) não foram observadas diferenças entre os valores de massa corporal medidos em homens adultos e os indicados por discentes iniciantes e concluintes do curso de Bacharelado em Educação Física, a partir da análise de fotografias daqueles homens. Esses dados em conjunto sugerem que a avaliação subjetiva da massa corporal a partir de fotografias de indivíduos apresenta complexidade diferente, de acordo com o sexo do avaliado, uma vez que a distribuição dos tecidos corporais (tecido adiposo e muscular) das mulheres adultas é diferente da dos homens adultos (BORKAN e HULTS, 1983; LOPES et al., 1995). Adicionalmente, parece que o nível de formação no curso de Educação física não influenciou diretamente na percepção da massa corporal de mulheres e homens adultos, a partir da análise de fotografias de corpo inteiro.

Apesar dos instrumentos utilizados no presente estudo (escala de STUNKARD et al., 1983 e as fotografias) apresentarem limitações, por mostrarem apenas uma visão bidimensional dos

Persp. online: biol. \& saúde, Campos dos Goytacazes, 30 (9),13- 23, 2019

seer.perspectivasonline.com.br 
contornos de corpos (MCCABE et al., 2006), os resultados referentes ao percentual de gordura não mostraram diferença entre os valores percebidos pelo G-AV1 e G-AV2 e nem quando os comparados comos valores de percentual de gordura estimados na avaliação antropométrica do GMOD. Diferentemente, Silva et al. (2018) observaram que os valores de percentual de gordura indicados por discentes iniciantes e concluintes do curso de Bacharelado em Educação Física foram maiores do que os valores de percentual de gordura estimados em homens adultos. Eles observaram ainda que os valores de percentual de gordura indicados pelos discentes iniciantes foram maiores do que os valores indicados pelos discentes concluintes. Sugerindo que, pelo menos para a avaliação do percentual de gordura a partir de fotografias de homens adultos, o processo de formação em Bacharel em Educação Física pode exercer alguma influência. Em conjunto, esses resultados reforçam a complexidade da avaliação da composição corporal, sobretudo a partir de fotografias de homens ou mulheres. Por outro lado, a reprodutibilidade das avaliações realizadas no presente estudo e no de Silva et al. (2018) favorecem a fidedignidade das mesmas.

Adicionalmente, é importante frisar que fatores como o número reduzido de discentes avaliadores e a não avaliação do ciclo menstrual das mulheres modelos [fator que pode interferir na percepção corporal (CARR-NANGLE et al., 1994)], constituem fatores limitantes à interpretação dos resultados.

Não sabemos ainda se os grupos de avaliadores fossem, também, divididos quanto ao sexo (masculino e feminino) encontraríamos os mesmos resultados. Mais estudos são necessários para avaliar-se sobre essa questão.

\section{CONCLUSÕES}

Levando-se em conta os resultados do estudo, concluímos que discentes ingressantes e concluintes do curso de Bacharelado em Educação Física da UFOP tiveram percepção semelhante sobre a imagem corporal, massa corporal e percentual de gordura, avaliados a partir de fotografias de mulheres adultas. Acredita-se que o processo de formação no curso de Bacharelado em Educação Física, por si só, não influencia de forma significativa a percepção da IC, massa corporal e percentual de gordura, avaliados a partir de fotografias de mulheres adultas. Sugerimos que estudos semelhantes com discentes de outras áreas de saúde sejam realizados para se compreender melhor essas questões.

\section{REFERÊNCIAS}

ÅLGARS M. et al. The adult body: How age, gender, and body mass index are related to body image. Journal of Aging Health, v.21, n.8, p.1112-1132, 2009. Disponível em: $<$ https://journals.sagepub.com>. Acesso em 05 ago 2017.

ASSUMPÇÃO, H. C. et al. Insatisfação com a imagem corporal em estudantes de educação física: comparação entre sexos. Coleção Pesquisa em Educação Física, v. 9, n. 2, p. 143-150, 2010.

AZEVEDO, A. P. et al. Dismorfia muscular: A busca pelo corpo hiper musculoso. Motricidade, v. 8, n. 1, p. 53-66, 2012. Disponível em: < http://www.scielo.mec.pt>. Acesso em 05 ago 2017.

BARRETT, L.; DUNBAR, R.; LYCETT, J. Human evolutionary psychology. Princeton, NJ: Princeton University Press, 2002. 448p. 
BORKAN, G. A.; HULTS, D. E. Change in body content and distribution during aging. American Journal of Physical Anthropology, v. 60, n. 2, p. 174-175, 1983.

BRASIL. Conselho Nacional de Educação. Câmara de Educação Superior. Resolução $\mathbf{n}^{\circ} \mathbf{6}$, de 18 de dezembro de 2018. Institui Diretrizes Curriculares Nacionais do Curso de Graduação em Educação Física e dá outras providências., Brasília, DF, dez, 2018.

CARR-NANGLE R. E. et al. Body image changes over the menstrual cycle in normal women. International Journal Eat Disorder, v. 16, n. 3, p. 267-273, 1994. Disponível em: $<$ https://www.researchgate.net>. Acesso em 08 jan 2019.

CASH T. F.; GREEN G. K. Body weight and body image among college women: Perception, cognition, and affect. Journal of Personality Assessment, v. 50, n. 2, p. 290-301, 1986.

COWAN N. What are the differences between long-term, short-term, and working memory? Progress in Brain Research, v. 169, p. 323-338, 2008.

CRAWFORD D.; CAMPBELL K. Lay definitions of ideal weight and overweight. International Journal of Obesity, v. 23, n. 7, p. 738-745, 1999. Disponível em: <https://www.researchgate.net>. Acesso em 23 jan 2019.

DAMASCENO V. O. et al. Tipo físico ideal e satisfação com a imagem corporal de praticantes de caminhada. Revista Brasileira de Medicina do Esporte, v. 11, n. 3, p. 181-186, 2005. Disponível em: <https://www.scielo.br>. Acesso em 04 jun 2017.

DAMASCENO V. O. et al. Imagem corporal e corpo Ideal. Revista Bras de Ciência e Movimento, v. 14, n. 2, p. 81-94, 2006. Disponível em: <https://www.researchgate.net>. Acesso em 12 jan 2019.

DAVISON, T. E.; MCCABE, M. P. Relationships between men's and women's body image and their psychological, social, and sexual functioning. Sex Roles, v. 7, n. 8, p. 463-475, 2005. Disponível em: <https://link.springer.com>. Acesso em 12 jan 2019.

FERNANDES T. C. et al. Aspectos da construção e desenvolvimento da imagem corporal e implicações na Educação Física. EFDeportes, Buenos Aires, v. 10, n. 3, 2005. Disponível em: $<$ https://www.efdeportes.com>. Acesso em: 23/05/2018.

FERRARI E. P.; SILVA D. A. S.; PETROSKI E. L. Associação entre percepção da imagem corporal e estágios de mudança de comportamento em acadêmicos de educação física. Revista Brasileira de Cineantropometria e Desempenho Humano, v. 14, n. 5, p. 535-544, 2010. Disponível em: <https://www.scielo.br>. Acesso em 07 jun 2017.

FERREIRA M. E. C.; DE CASTRO A. P. A.; GOMES G. A obsessão masculina pelo corpo: malhado, forte e sarado. Revista Brasileira de Ciência do Esporte, v. 27, n. 1, p. 167-182, 2005. Disponível em: <http://revista.cbce.org.br>. Acesso em 23 jan 2019.

FISHER S. et al. Comparison of body image evaluation by virtual reality and paper-based figure rating scales in adolescents with anorexia nervosa: retrospective study. Eating and Weight Disord, p. 1-9, 2019. Disponível em: <https://link.springer.com>. Acesso em 03 maio 2019.

FLORIANO, J. M.; D’ALMEIDA, K. S. Prevalência de transtorno dismórfico muscular em homens adultos residentes na fronteira oeste do Rio Grande do Sul. Revista Brasileira de 
Nutrição Esportiva, v. 10, n. 58, p. 448-457, 2016. Disponível em: <http://www.rbne.com.br>. Acesso em 07 jun 2018.

GARNER D. M. et al. Cultural expectations of thinness in women. Psychological Reports, v. 47, n. 2, p.483-491, 1980.

GIORDANI, R. C. F. A auto-imagem corporal na anorexia nervosa: uma abordagem sociológica. Psicologia \& Sociedade, v. 18, n. 2, p. 81-88, 2006. Disponível em: $<$ https://www.scielo.br>. Acesso em 07 set 2016.

JACKSON A. S.; POLLOCK M. L.; WARD A. Generalized equations for predicting body density of women. Medicine and Science in Sports and Exercise, v. 12, n. 3, p. 175-182, 1980.

KAMEL E. G.; MCNEILL G. Men are less aware of being overweight than women. Obesity Research, Baton Rouge, v. 8, n. 8, p. 604, 2000.

KAY S. The psychological and anthropometric in body image. In: Norton K, Olds T, eds. Anthropometrica. Sydney, Austrália: University of New South Wales Press 1996, p. 217-238.

LOPES, A. S. et al. Distribuição da gordura subcutânea e índices de adiposidade de 20 a 67 anos de idade. Revista Brasileira de Atividade Física e Saúde, v. 1, n. 2, p. 15-26, 1995. Disponível em: <http://rbafs.org.br>. Acesso em $05 \mathrm{dez} 2018$.

MARTINS C. R. et al. Insatisfação com a imagem corporal e relação com estado nutricional, adiposidade corporal e sintomas de anorexia e bulimia em adolescentes. Revista de Psiquiatria do Rio Grande do Sul, Porto Alegre, v. 32, n. 1, p. 19-23, 2010. Disponível em: $<$ https://www.scielo.br>. Acesso em 07 set 2016.

MCCABE M. P. et al. Accuracy of body size estimation: Role of biopsychosocial variables. Body Image, v. 3, n. 2, p. 163-171, 2006. Disponível em: <https://www.sciencedirect.com>. Acesso em 07 set 2016.

MEDEIROS T. H.; CAPUTO E. L.; DOMINGUES M. R. Insatisfação corporal em frequentadoras de academia. Jornal Brasileiro de Psiquiatria, Rio de Janeiro, v. 66, n. 1, p. 38-44, 2017. Disponível em: <https://www.scielo.br>. Acesso em 04 jun 2018.

RAMIREZ E. M.; ROSEN J. C. A comparison of weight control and weight control plus body image therapy for obese men and women. Journal of Consulting and Clinical Psychology, Washington, v. 69, n. 3, p. 440-446, 2001.

SILVA D. M. et al. Percepção Subjetiva de Alunos do Curso de Educação Física sobre a Imagem Corporal e Variáveis Antropométricas de Homens Adultos. Revista Brasileira de Ciências da Saúde, v. 22, n. 3, p. 213-220, 2018. Disponível em: <http://www.periodicos.ufpb.br>. Acesso em 30 dez 2018.

SIRI W. E. Body composition from fluid space and density. In: Brozek J, Hanschel A, eds. Techniques for measuring body composition. Washington, DC: National Academy of Science 1961, p. 223-244.

STUNKARD A. J.; SØRENSEN T.; SCHULSINGER F. Use of the Danish Adoption Register for the study of obesity and thinness. Research publications - Association for Research in Nervous and Mental Disease, v. 60, p. 115-120, 1983. 